\title{
GIRRT Motion Planning Algorithm for Humanoid Robot
}

\author{
Hua-Zhong $\mathrm{LI}^{1, \text { a }}$, Zhuo LIANG ${ }^{2, \mathrm{~b}}$ \\ ${ }^{1}$ Software Department, Shenzhen Institute of Information Technology, Shenzhen, 518029, China \\ ${ }^{2}$ Shenzhen Middle School, Shenzhen, 518025, China \\ alihz@sziit. edu.cn, ${ }^{\text {b2 } 2862001893 @ q q . c o m ~}$
}

Keywords: humanoid robot; manipulation; rapidly-exploring random tree; generalized inverse

\begin{abstract}
Aiming at such problems as unknown goal configuration, grasp space limitation, a large number of barriers in configuration space and high-dimension space redundancy during planning motion of humanoid robot arms grasping target, this paper proposes a generalized inverse rapid-exploring random tree (GIRRT) collision avoidance method. Firstly, it establishes kinematics and inverse kinematics models for upper arm of humanoid robot based on hierarchical sub-dimension space motion planning concept model. Secondly, it proposes a weighted minimal norm GIRRT planning algorithm. Finally, the effectiveness of the algorithm proposed in this paper has been verified via computer 3D simulation.
\end{abstract}

\section{Introduction}

Motion planning of humanoid robot arms grasping space object is about the motion planning ${ }^{[1-2]}$ with multiple constraints in a typical high-dimension C-space. This is a challenging frontier research field. Aiming at the complex system of close coupling humanoid robot, the complex problems of high-dimension space motion planning for humanoid robot are divided into a series of sub-problems in low-dimension space. Constraint coordinate scheme is applied to solve the sub-problems with increment mode ${ }^{[3-5]}$.

At present, high-dimension space planning includes two typical methods: the methods based on random sampling, such as Probabilistic Road Map (PRM) ${ }^{[6-8]}$ and Rapidly-exploring Random Tree $(\mathrm{RRT})^{[9-11]}$. Kuffner et al succeeded in applying RRT method in biped walking robot H5 to solve walking, pose transformation, grasp, manipulation and motion planning ${ }^{[12-13]}$. As for joint-type humanoid robot structure lacking kinematics analytical solution, this paper adopts the motion planning method based on random sampling to provide humanoid robot motion planning with new solutions according to the heuristic information provided by the robot in end-effector pose status in work space. Bertram et al applied the distance measurement of end-effector of humanoid robot in work space opposite to goal configuration as the heuristic information of Configuration Space (C-space) motion planning and guided RRT algorithm to rapidly expand towards the goal configuration in C-space ${ }^{[14]}$. Weghe et al applied Jacobian matrix transposition method to directly transform the heuristic information in work space into joint space configuration and improve the performance of humanoid robot motion planning ${ }^{[15]}$. Berenson et al applied projection technology to propose restricted bi-direction RRT algorithm and move sampling waypoints to constraint manifold and realize rapid motion planning for mechanical arm grasp with 7 DoFs ${ }^{[16]}$. When the goal configuration of humanoid robot arms is unknown, inverse kinematics solver is needed to guide RRT to expand towards the goal. The method based on transposed Jacobian matrix can be used to generate C-space and avoid seeking inverse kinematics (IK) solution. When the analytical solution of robot IK cannot be obtained and only goal grasp pose is known, RRT method based on transposed Jacobian matrix will be very useful. Apparently, double-tree or multiple-tree RRT cannot be realized due to the lack of specific C-space goal pose.

Aiming at motion planning problems of humanoid robot arms grasping objects, this paper firstly proposes a motion planning concept model for hierarchical sub-dimension space. Then, it further studies the kinematics and inverse kinematical modeling of humanoid robot arms. Based on this, it proposes a weighted minimal norm GIRRT planning algorithm. Finally, the effectiveness of the 
algorithm proposed in this paper has been verified via computer 3D visualization simulation.

\section{Problem Description}

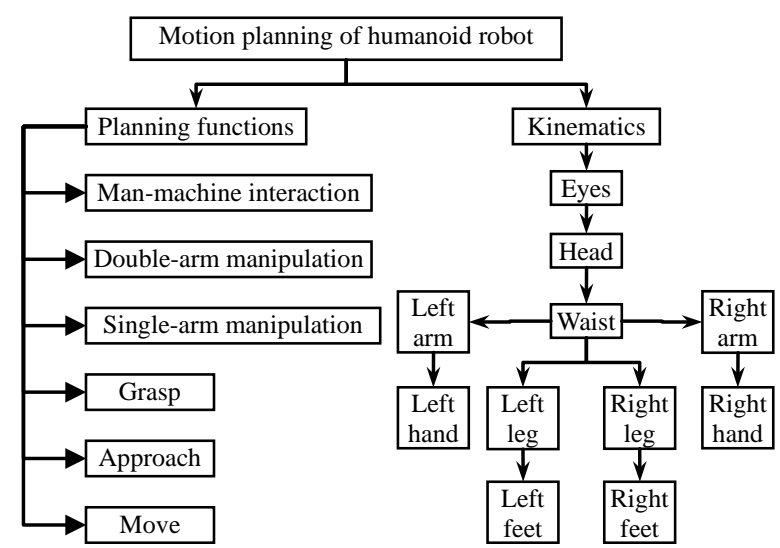

Fig.1 Motion planning concept model for hierarchical sub-dimension space of humanoid robot

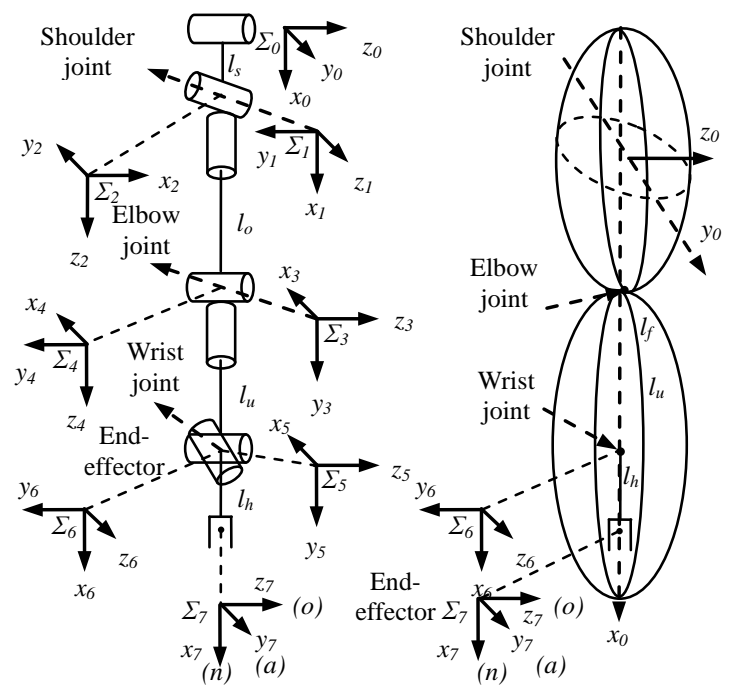

Fig.2 Diagram for right arm model of humanoid robot and work space

The arm grasp motion planning studied in this paper can be set as: keep the joints of lower limbs (left and right feet, left and right legs) still, and complete goal grasp and other operations by only depending on cooperation and collaboration of waist, left and right arms.

Suppose kinematic chain of humanoid robot consisting of waist and left (right) arms is marked as $\left\{A^{0}, A^{1}\right\}$, where $A^{0}$ is the root of kinematic chain including 3 rotational DoF--yaw angle $\alpha_{0}$, pitch angle $\beta_{0}$ and rolling angle $\gamma_{0}$ for waist joint motion, which is expressed as $\mathbf{q}_{0}=\left[\alpha_{0}, \beta_{0}, \gamma_{0}\right]^{T} \equiv\left[\theta_{1}, \theta_{2}, \theta_{3}\right]^{T}$. Left (right) arm $A^{1}$ includes 7 DoFs such as shoulder, elbow and wrist, which is expressed as $\mathbf{q}_{1}=\left[q_{1}, \cdots, q_{7}\right]^{T} \equiv\left[\theta_{4}, \cdots, \theta_{10}\right]^{T}$. The number of DoF $n$ is 10 . Its configuration can be expressed as $\boldsymbol{\theta}=\left[\mathbf{q}_{0}^{T}, \mathbf{q}_{1}^{T}\right]^{T} \equiv\left[\theta_{1}, \cdots, \theta_{10}\right]^{T}$, initial configuration is $\boldsymbol{\theta}_{\text {start }}=\left[\theta_{1 \text { start }}, \cdots, \theta_{10 \text { start }}\right]^{T}$, and goal configuration is $\mathbf{p}_{\text {obj }} \in \mathbf{R}^{6}$. Feasible grasp set is $\mathbf{g}_{c}=\left\{g_{0}, \ldots, g_{N_{r}}\right\}$, and forward kinematical equation is $\mathbf{p}_{e}=\mathbf{f}(\boldsymbol{\theta})$. Then, the motion planning for humanoid robot arm grasping object studied in this paper can be defined as:

Given that the initial configuration of the robot is $\boldsymbol{\theta}_{\text {start }} \in \mathbf{C}_{\text {free }}$ (free C-space), its object configuration is $\mathbf{p}_{\text {obj }}$ and feasible set is $\mathbf{g}_{c}$. Its goal configuration $\boldsymbol{\theta}_{\text {goal }}$ is unknown. Find continuous path mapping in C-space which can avoid collision, singularity and meet joint constraint conditions: $\tau:[0,1] \rightarrow \mathbf{C}_{\text {free }}$ so as to obtain $\tau[0]=\boldsymbol{\theta}_{\text {start }}, \quad \tau[1]=\boldsymbol{\theta}_{\text {goal }}, \quad\left(\mathbf{p}_{\text {obj }}, \mathbf{g}_{c}\right) \rightarrow \mathbf{f}\left(\boldsymbol{\theta}_{\text {goal }}\right)$.

\section{Kinematical Modeling of Humanoid Robot}

\section{Kinematic Model for Arms}

Reference coordinate system of right arm with 7DoFs of humanoid robot based on D-H, coordinate system of connecting rod and work space diagram is shown in Fig.2. Its D-H parameter is shown in Table 1. Suppose $\mathbf{q}_{1}=\left[\begin{array}{lll}q_{1}, & \ldots, & q_{7}\end{array}\right]^{T} . \sin q_{i}$ and $\cos q_{i}$ are respectively marked as $s_{q_{i}}$ and $c_{q_{i}}$, and abbreviated as $s_{i}$ and $c_{i}$. Then, the homogeneous matrix ${ }^{i-1} \mathbf{T}_{i}\left(q_{i}\right)$ between coordinate systems $\mathrm{i}$ and $\mathrm{i}-1$ can be expressed as Formula (1): 
Table $1 \mathrm{D}-\mathrm{H}$ parameters of right arm of humanoid

\begin{tabular}{|c|c|c|c|c|c|}
\hline $\mathrm{i}$ & $\mathrm{q}_{\mathrm{i}}$ (radian) & $\alpha_{\mathrm{i}}$ (radian) & $\mathrm{a}_{\mathrm{i}}(\mathrm{mm})$ & $\mathrm{d}_{\mathrm{i}}(\mathrm{mm})$ & Joint constraint \\
\hline 1 & $\mathrm{q}_{1}$ & $-\pi / 2$ & $l_{\mathrm{S}}$ & 0 & {$[-\pi / 2, \pi / 2]$} \\
\hline 2 & $\mathrm{q}_{2}-\pi / 2$ & $-\pi / 2$ & 0 & 0 & {$[-\pi / 2, \pi / 2]$} \\
\hline 3 & $\mathrm{q}_{3}+\pi / 2$ & $\pi / 2$ & 0 & $l_{u}$ & {$[-23 \pi / 18, \pi / 2]$} \\
\hline 4 & $\mathrm{q}_{4}$ & $-\pi / 2$ & 0 & 0 & {$[0,29 \pi / 36]$} \\
\hline 5 & $\mathrm{q}_{5}$ & $\pi / 2$ & 0 & $l_{f}$ & {$[0,35 \pi / 18]$} \\
\hline 6 & $\mathrm{q}_{6}+\pi / 2$ & $-\pi / 2$ & 0 & 0 & {$[-\pi / 4, \pi / 4]$} \\
\hline 7 & $\mathrm{q}_{7}$ & $\pi / 2$ & $l_{h}$ & 0 & {$[-\pi / 4, \pi / 4]$} \\
\hline & $\begin{array}{c}-c_{\alpha_{i}} S_{i} \\
C_{\alpha_{i}} C_{i} \\
S_{\alpha_{i}} \\
0\end{array}$ & $\left.\begin{array}{c}a_{i} c_{i} \\
a_{i} s_{i} \\
d_{i} \\
1\end{array}\right]$ & & & \\
\hline
\end{tabular}

Forward kinematical (FK) equation for right arm model of humanoid robot can be described by end-effector $\Sigma_{7}$ pose of right arm opposite to homogeneous coordinate transformation matrix of reference coordinate system $\Sigma_{0}$ as:

$$
{ }^{0} \mathbf{T}_{7}\left(\mathbf{q}_{1}\right)=\prod_{i=1}^{7}{ }^{i-1} \mathbf{T}_{i}\left(q_{i}\right)=\left[\begin{array}{cccc}
\mathbf{n} & \mathbf{0} & \mathbf{a} & \mathbf{p} \\
0 & 0 & 0 & 1
\end{array}\right]
$$

In Formula (2), the right arm of robot is of redundancy. The rotational description that the elbow joint center surrounds the wrist joint and $\Sigma_{0}$ original point axis. Once the pose of elbow joint is determined, the analytical form for arm inverse kinematics can be deduced. According to the elbow circle of right arm shown in Fig.3, the pose of arm elbow pose ${ }^{0} \mathbf{T}_{4}$ can be calculated.

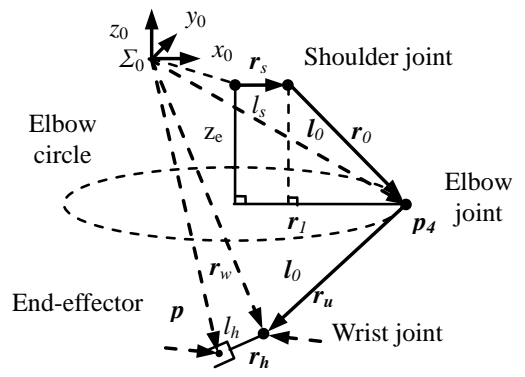

Fig.3 Calculation of elbow joint pose of right hand

$$
{ }^{0} \mathbf{T}_{4}=\prod_{i=1}^{4}{ }^{i-1} \mathbf{T}_{i}\left(q_{i}\right)=\left[\begin{array}{cccc}
\mathbf{n}_{4} & \mathbf{o}_{4} & \mathbf{a}_{4} & \mathbf{p}_{4} \\
0 & 0 & 0 & 1
\end{array}\right]
$$

Where, $\mathbf{a}_{4}=\mathbf{r}_{w}-\mathbf{p}_{4} /\left\|\mathbf{r}_{w}-\mathbf{p}_{4}\right\|, \quad \mathbf{r}_{w}=\mathbf{p}-l_{h} \cdot \mathbf{n}, \mathbf{o}_{4}=\mathbf{a}_{4} \times\left(\mathbf{r}_{s}-\mathbf{p}_{4}\right) /\left\|\mathbf{a}_{4} \times\left(\mathbf{r}_{s}-\mathbf{p}_{4}\right)\right\|, \mathbf{n}_{4}=\mathbf{o}_{4} \times \mathbf{a}_{4}$.

The pose of end-effector of right arm opposite to the right elbow joint is:

$$
{ }^{4} \mathbf{T}_{7}=\prod_{i=5}^{7}{ }^{i-1} \mathbf{T}_{i}\left(q_{i}\right)=\prod_{i=4}^{1}{ }^{i-1} \mathbf{T}_{i}^{-1}\left(q_{i}\right) \cdot{ }^{0} \mathbf{T}_{4}=\left[\begin{array}{cccc}
\mathbf{n}_{e} & \mathbf{o}_{e} & \mathbf{a}_{e} & \mathbf{p}_{e} \\
0 & 0 & 0 & 1
\end{array}\right]
$$

Thus, the inverse kinematics problems of right arm with 7DoFs can be decomposed into Formula (3) and Formula (4) to express sub-inverse kinematics problems of two smaller dimensions. According to Formula (3), multiply ${ }^{0} \mathbf{T}_{1}^{-1}$ on the two sides of the formula to obtain:

$$
{ }^{0} \mathbf{T}_{1}^{-1}\left(q_{1}\right) \cdot{ }^{0} \mathbf{T}_{4}=\prod_{i=2}^{4}{ }^{i-1} \mathbf{T}_{i}\left(q_{i}\right)
$$

The following formulas can be obtained from the equation consisting of the matrix $(3,4)$ and other elements on the two sides of Formula (5):

$$
\begin{aligned}
& q_{1} \equiv \theta_{4}=\operatorname{atan} 2\left( \pm p_{4 y}, \pm p_{4 x}\right) \\
& q_{2} \equiv \theta_{5}=\operatorname{atan} 2\left(-p_{4 z}, c_{1} p_{4 x}+s_{1} p_{4 y}-l_{s}\right)
\end{aligned}
$$

Multiply the inverse matrix ${ }^{2} \mathbf{T}_{1}^{-1}\left(q_{2}\right)$ on the two sides of Formula (5) to obtain: 


$$
\prod_{i=2}^{1}{ }^{i-1} \mathbf{T}_{i}^{-1}\left(q_{i}\right) \cdot{ }^{0} \mathbf{T}_{4}=\prod_{i=3}^{4}{ }^{i-1} \mathbf{T}_{i}\left(q_{i}\right)
$$

Compare the matrixes on the two sides of Formula (8) to obtain $q_{3}$ and $q_{4}$ :

$$
\begin{aligned}
& q_{3}=\operatorname{atan} 2\left(-s_{1} O_{4 x}+c_{1} O_{4 y},-s_{2} c_{1} O_{4 x}-s_{2} s_{1} O_{4 y}-c_{2} O_{4 z}\right) \\
& q_{4} \equiv \theta_{7} \operatorname{atan} 2\left(c_{2} c_{1} n_{4 x}+c_{2} s_{1} n_{4 y}-s_{2} n_{4 z}, \quad c_{2} c_{1} a_{4 x}+c_{2} s_{1} a_{4 y}-s_{2} a_{4 z}\right)
\end{aligned}
$$

It can be obtained from Formula (4):

$$
{ }^{4} \mathbf{T}_{5}^{-1}\left(q_{i}\right) \cdot{ }^{4} \mathbf{T}_{7}=\prod_{i=6}^{7}{ }^{i-1} \mathbf{T}_{i}\left(q_{i}\right)
$$

The following formulas can be obtained from the equation consisting of the matrix and other elements on the two sides of Formula (11):

$$
\begin{aligned}
& q_{5} \equiv \theta_{8}=\operatorname{atan} 2\left( \pm o_{e y}, \pm o_{e x}\right) \\
& q_{6} \equiv \theta_{9}=\operatorname{atan} 2\left(-o_{e z},-c_{5} o_{e x}-s_{5} o_{e y}\right) \\
& q_{7} \equiv \theta_{10}=\operatorname{atan} 2\left(-s_{5} n_{e x}+c_{5} n_{e y}, s_{5} a_{e x}-c_{5} a_{e y}\right)
\end{aligned}
$$

According to Formulas (6), (7), (9), (11), (12), (13) and (14), IK analytical equation for the right arm of robot can be obtained. Similarly, the forward and inverse kinematical equations for left arm of humanoid robot can be obtained.

\section{Kinematic model for waist}

The motion of the waist of humanoid robot in 3D space opposite to inertial reference system $\Sigma_{I}$ in lower limbs can be expressed with $4 \times 4$ homogeneous coordinate matrix ${ }^{I} \mathbf{T}_{0}\left(\alpha_{0}, \beta_{0}, \gamma_{0}\right)$ as $\equiv^{I} \mathbf{T}_{0}\left(\theta_{1}, \theta_{2}, \theta_{3}\right)$, including translation constant $\mathbf{p}_{t}=\left[x_{t}, y_{t}, z_{t}\right]^{T}$ in 3 directions and rotation around 3 axes which are expressed by yaw angle $\alpha_{0}$, pitch angle $\beta_{0}$ and rolling angle $\gamma_{0}$. Wherein, yaw angle $\alpha_{0}$ is a rotation angle around $\mathrm{z}$ axis, and its rotation matrix $\mathbf{R}_{z}\left(\alpha_{0}\right)$ can be expressed as:

$$
\mathbf{R}_{z}\left(\alpha_{0}\right)=\left[\begin{array}{ccc}
c_{\alpha_{0}} & -s_{\alpha_{0}} & 0 \\
s_{\alpha_{0}} & c_{\alpha_{0}} & 0 \\
0 & 0 & 1
\end{array}\right] \equiv \mathbf{R}_{z}\left(\theta_{1}\right)
$$

$\beta_{0}$ is a rotation angle around y axis, and its rotation matrix $\mathbf{R}_{y}\left(\beta_{0}\right)$ can be expressed as:

$$
\mathbf{R}_{y}\left(\beta_{0}\right)=\left[\begin{array}{ccc}
c_{\beta_{0}} & 0 & s_{\beta_{0}} \\
0 & 1 & 0 \\
-s_{\beta_{0}} & 0 & c_{\beta_{0}}
\end{array}\right] \equiv \mathbf{R}_{y}\left(\theta_{2}\right)
$$

$\gamma_{0}$ is a rotation angle around $\mathrm{x}$ axis, and its rotation matrix $\mathbf{R}_{x}\left(\gamma_{0}\right)$ can be expressed as:

$$
\mathbf{R}_{x}\left(\gamma_{0}\right)=\left[\begin{array}{ccc}
1 & 0 & 0 \\
0 & c_{\gamma_{0}} & -s_{\gamma_{0}} \\
0 & s_{\gamma_{0}} & c_{\gamma_{0}}
\end{array}\right] \equiv \mathbf{R}_{x}\left(\theta_{3}\right)
$$

Rotation combination can be expressed as:

$$
\begin{aligned}
& \mathbf{R}\left(\theta_{1}, \theta_{2}, \theta_{3}\right)=\mathbf{R}_{z}\left(\theta_{1}\right) \mathbf{R}_{y}\left(\theta_{2}\right) \mathbf{R}_{x}\left(\theta_{3}\right) \\
& \text { Then, }{ }^{I} \mathbf{T}_{0}\left(\theta_{1}, \theta_{2}, \theta_{3}\right)=\left[\begin{array}{cc}
\mathbf{R}\left(\theta_{1}, \theta_{2}, \theta_{3}\right) & \mathbf{p}_{t} \\
0 & 1
\end{array}\right]
\end{aligned}
$$

According to Formula (1) and Formula (19), homogeneous transformation matrix ${ }^{I} \mathbf{T}_{7}(\boldsymbol{\theta})$ for end-effector coordinate system $\Sigma_{e}\left(\equiv \Sigma_{7}\right)$ opposite to inertial coordinate system $\Sigma_{I}$ can be obtained:

$$
{ }^{I} \mathbf{T}_{7}(\boldsymbol{\theta})={ }^{I} \mathbf{T}_{0}\left(\theta_{1}, \theta_{2}, \theta_{3}\right) \cdot{ }^{0} \mathbf{T}_{7}\left(\mathbf{q}_{1}\right)
$$

According to Formula (20), forward kinematics relation for kinematic chain joint 
configuration $\boldsymbol{\theta}$ and end-effector pose $\mathbf{p}_{e}$ formed from the waist of humanoid robot and arm end-effector:

$$
\mathbf{p}_{e}=\mathbf{f}(\boldsymbol{\theta})
$$

\section{WLN Generalized Inverse}

Suppose the pose and velocity of end-effector of humanoid robot in Cartesian space $\operatorname{are} \mathbf{p}_{e}$ and $\dot{\mathbf{p}}_{e} \in \mathbf{R}^{6 \times 1}$, and its angle position and velocity in joint space are $\boldsymbol{\theta}$ and $\dot{\boldsymbol{\theta}} \in \mathbf{R}^{10 \times 1}$. According to Formula (21), the forward kinematics differential equation for humanoid robot can be obtained:

$$
\dot{\mathbf{p}}_{e}=\mathbf{J}(\boldsymbol{\theta}) \dot{\boldsymbol{\theta}}
$$

Where, $\mathbf{J}(\boldsymbol{\theta}) \in \mathbf{R}^{6 \times 10}$ is Jacobian matrix for humanoid robot arms. The inverse kinematics solution for redundancy kinematical equation (22) is:

$$
\dot{\boldsymbol{\theta}}=\mathbf{J}^{+} \dot{\mathbf{p}}_{e}+\left(\mathbf{I}-\mathbf{J}^{+} \mathbf{J}\right) \dot{\boldsymbol{\xi}}
$$

Where, $\mathbf{J}^{+}(\boldsymbol{\theta})=\mathbf{J}^{T}\left(\mathbf{J J}^{T}\right)^{-1}$ is Moore-Penrose generalized inverse matrix of Jacobian matrix $\mathbf{J}$. It can be calculated with singular value decomposition method. $\mathbf{I} \in \mathbf{R}^{10 \times 10}$ is unit matrix, $\dot{\xi} \in \mathbf{R}^{10}$ is random vector, and $\mathbf{J}^{+} \dot{\mathbf{p}}_{e}$ is particular solution for joint velocity which defined the movement of humanoid robot. $\left(\mathbf{I}-\mathbf{J}^{+} \mathbf{J}\right) \dot{\xi}$ is homogeneous solution. There is no motion in end-effector.

According to typical gradient projection method ${ }^{[17]}$ proposed by Liegeois, use $\mathbf{k} \nabla \mathbf{H}(\boldsymbol{\theta})$ to replace $\dot{\xi}$. Formula (23) can be rewritten as:

$$
\dot{\boldsymbol{\theta}}=\mathbf{J}^{+} \dot{\mathbf{p}}_{e}+k\left(\mathbf{I}-\mathbf{J}^{+} \mathbf{J}\right) \nabla \mathbf{H}(\boldsymbol{\theta})
$$

Where, $\nabla \mathbf{H}(\boldsymbol{\theta})=\frac{\partial \mathbf{H}(\boldsymbol{\theta})}{\partial \boldsymbol{\theta}}=\left[\begin{array}{lll}\frac{\partial \mathbf{H}}{\partial \theta_{1}} & \cdots & \frac{\partial \mathbf{H}}{\partial \theta_{10}}\end{array}\right]^{T}$ is the gradient of optimizing index $\mathbf{H}(\boldsymbol{\theta})$ and scalar real constant $k$ is automatic amplification coefficient. Thus, Formula (24) can be obtained from the sum of minimum norm solution and optimization item:

$$
\dot{\boldsymbol{\theta}}=\dot{\boldsymbol{\theta}}_{\text {min }}+k \nabla \mathbf{H}(\boldsymbol{\theta})
$$

Where, the minimum norm solution $\dot{\boldsymbol{\theta}}_{\text {min }}=\dot{\boldsymbol{\theta}}_{p}-\left[\frac{\dot{\boldsymbol{\theta}}_{p}^{T} \dot{\boldsymbol{\theta}}_{h}}{\dot{\boldsymbol{\theta}}_{h}^{T} \dot{\boldsymbol{\theta}}_{h}}\right] \dot{\boldsymbol{\theta}}_{h}, \dot{\boldsymbol{\theta}}_{p}=\left[\begin{array}{c}\mathbf{0} \\ \mathbf{J}_{M}^{-1}\left(\dot{\mathbf{p}}_{e}-k \mathbf{J} \nabla \mathbf{H}\right)\end{array}\right]$ (a particular solution for $\dot{\boldsymbol{\theta}}$ ) and $\dot{\boldsymbol{\theta}}_{h}=\left[\begin{array}{c}\mathbf{1} \\ \mathbf{J}_{M}^{-1} \mathbf{J}_{\boldsymbol{a}}\end{array}\right]$ (homogeneous solution for $\dot{\boldsymbol{\theta}}$ ). $\mathbf{J}_{M} \in \mathbf{R}^{6 \times 6}$ is $6 \times 6$ nonsingular matrix after 4 columns are taken by $\mathbf{J}$. $\mathbf{J}_{\boldsymbol{\alpha}} \in \mathbf{R}^{6 \times 4}$ is the matrix after 4 columns are divided by $\mathbf{J}$.

For joint displacement ultralimit in the process of grasp operation of humanoid robot, $\mathbf{H}(\boldsymbol{\theta})$ is taken as:

$$
\mathbf{H}(\boldsymbol{\theta})=\sum_{i=1}^{10} \frac{\left(\theta_{i \max }-\theta_{i \min }\right)^{2}}{4 \cdot\left(\theta_{i \max }-\theta_{i}\right)\left(\theta_{i}-\theta_{i \min }\right)}
$$

According to damping Weighted Least-Norm (WLN) method, the optimal solution for joint velocity of humanoid robot is:

$$
\dot{\boldsymbol{\theta}}=\mathbf{J}_{W L N}^{+}(\boldsymbol{\theta}) \cdot \dot{\mathbf{p}}_{e}
$$

Where, $\mathbf{J}_{W L N}^{+}(\boldsymbol{\theta})=\mathbf{W}^{-1} \mathbf{J}^{T}\left[\mathbf{J} \cdot \mathbf{W}^{-1} \mathbf{J}^{T}\right]^{-1}$. Based on $\mathbf{H}(\boldsymbol{\theta}), \mathbf{W}(i=1 \sim 10)$ can be obtained:

$$
\mathbf{W}=\left[\begin{array}{cccc}
w_{1} & 0 & \cdots & 0 \\
0 & w_{2} & \cdots & 0 \\
\vdots & \vdots & \ddots & \vdots \\
0 & 0 & \cdots & w_{10}
\end{array}\right], w_{i}=\left\{\begin{array}{cc}
1+\left|\partial \mathbf{H}(\boldsymbol{\theta}) / \partial \theta_{i}\right| & \text { if } \Delta\left|\partial \mathbf{H}(\boldsymbol{\theta}) / \partial \theta_{i}\right| \geq 0 \\
1 & \text { other }
\end{array}\right.
$$




\section{WLN GIRRT Algorithm}

Aiming at the grasp task by the arms of humanoid robot, this paper proposes a weighted least-norm (WLN) GIRRT planning algorithm. The pseudo code for this algorithm can be described as:

Algorithm 1: WLNGenInvRRT( $\left.\boldsymbol{\theta}_{\text {start }}, \mathbf{p}_{\text {obj }}, \mathbf{g}_{c}\right)\{$

1: RRT.SetSConfig $\left(\boldsymbol{\theta}_{\text {start }}\right) / /$ set initial configuration

2: do $\{/ /$ execute single-tree RRT motion planning

3: NormalExtend $=$ true $/ /$ set random extension tab

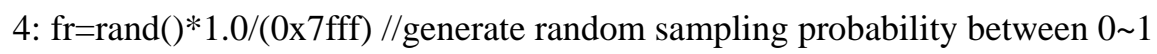

5: if $(\mathrm{fr} \leq \mathrm{fExtend})$ \{//extend to grasp goal configuration

6: $\quad$ NormalExtend $=$ false //indicate extension to goal configuration

7: ExtendStatus=ExtendToGrasp(RRT, ${ }_{\text {obj }}, \mathbf{g}_{c}$ )

8:if (ExtendStatus $==$ RRT_ERROR) \{

9: $\quad$ StopSearch $=$ true $\} / /$ set planning stop tab

10:if(ExtendStatus==RRT_REACHED) $\{/ /$ reach goal

11: RRT.GetConfig( ${ }^{\boldsymbol{\theta}_{\text {goal }}}$ )//get goal configuration

12: RRT.GetSolutionGrasp() //get grasp goal solution

13: FoundSolution=true//find path solution $\}$ \}

14: if ( NormalExtned ) \{

15: RRT.RandomConfig( $\left.\boldsymbol{\theta}_{\text {rand }}\right)$

16: $\quad \boldsymbol{\theta}_{\text {near }}=$ RRT.NearestNeighbor $\left(\boldsymbol{\theta}_{\text {rand }}\right)$

17: ExtendStatus $=$ RRT.Connect( $\left(\boldsymbol{\theta}_{\text {near }}, \boldsymbol{\theta}_{\text {rand }}, \boldsymbol{\theta}_{\text {new }}\right)$

18:if (ExtendStatus==RRT_ERROR)

$\{$ StopSearch=true $\}$ \}

19: $\quad$ Cycles $++/ /$ search times +1

20: $\quad\}$ while (!StopSearch \&\& Cycles $<$ MaxCycles $\& \&$ !FoundSolution)

21: return RRT.GetSolutionPath();

Algorithm description: Set goal extension probability $f_{\text {Extend }}$ as 0.3 . The maximum cyclic search times are set as MaxCycles $=40000$. According to even distribution probability, RandomConfig( $\left.{ }^{\boldsymbol{\theta}_{\text {rand }}}\right)$ conducts random sampling in C-space to obtain $\boldsymbol{\theta}_{\text {rand }}=\boldsymbol{\theta}_{\min }+\operatorname{rand}() *$ (1/ $0 \mathrm{x} 7 \mathrm{fff}) *\left(\boldsymbol{\theta}_{\max } \boldsymbol{\theta}_{\text {min }}\right)$. The function of NearestNeighbor $\left(\boldsymbol{\theta}_{\text {rand }}\right)$ is to find the configuration $\boldsymbol{\theta}_{\text {near }}$ which is nearest to $\boldsymbol{\theta}_{\text {rand }}$. That is, $\min \left\|\boldsymbol{\theta}_{\text {near }}-\boldsymbol{\theta}_{\text {rand }}\right\|^{2}=\min \sum_{i=1}^{10}\left(\boldsymbol{\theta}_{\text {near }, i}-\boldsymbol{\theta}_{\text {rand }, i}\right)^{2}$. Connect $\left(\boldsymbol{\theta}_{\text {near }}, \boldsymbol{\theta}_{\text {rand }}, \boldsymbol{\theta}_{\text {new }}\right)$ is to realize the extension from the step length $\lambda$ to $\boldsymbol{\theta}_{\text {rand }}$ until any barrier is met. The new configuration $\boldsymbol{\theta}_{\text {near }}$ is obtained. Its operating principle diagram is shown in Fig.4.

ExtendToGrasp(RRT, $\mathbf{p}_{o b j}, \mathbf{g}_{c}$ ) is the core algorithm which extends towards the goal configuration by applying WLN generalized inverse. Its pseudo code is described as: 


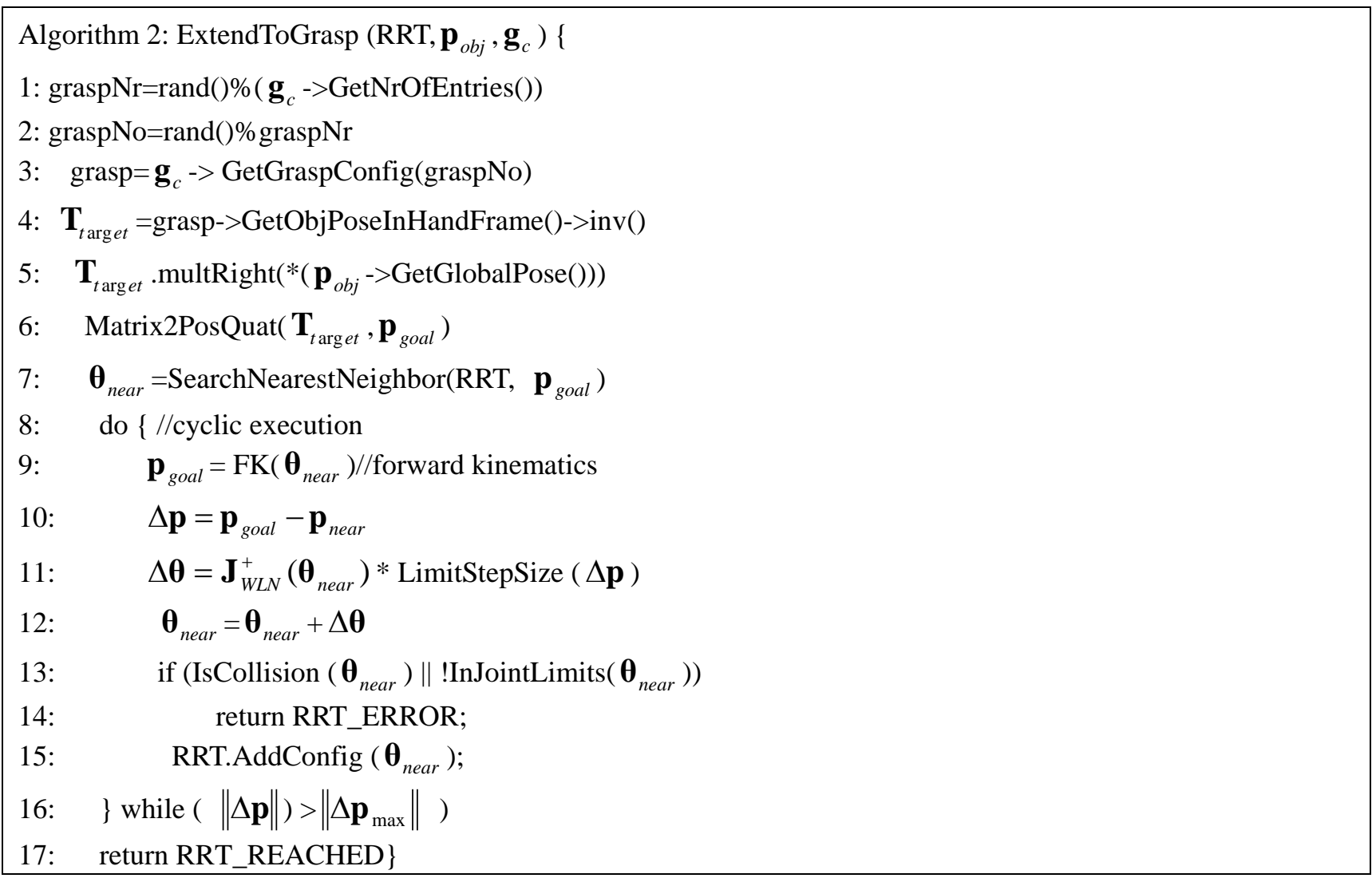

Algorithm description: Code 1-3 lines finishes the function of randomly selecting configuration from grasp set $\mathbf{g}_{c}$. According to grasp configuration and object configuration $\mathbf{p}_{o b j}$, code 4-6 lines calculate homogeneous transformation matrix $\mathbf{T}_{\text {target }}$ and configuration vector $\mathbf{p}_{\text {goal }}$ for end-effector of the arms of humanoid robot. According to forward kinematics formula (21), codes 9 lines calculate the end-effector configuration ${ }^{\mathbf{p}_{\text {goal }}}$ of humanoid robot at the configuration $\boldsymbol{\theta}_{\text {near }}$. According to WLN generalized velocity increment solution formula (27), code 11 lines calculate $\Delta \boldsymbol{\theta}$.

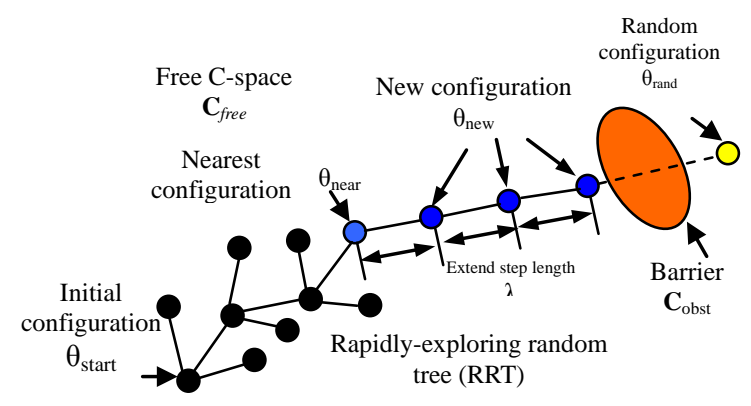

Fig.4 Diagram for Connect Principle

\section{Computer Visualization Simulation}

Taking humanoid robot Armar III left arm avoiding multiple barriers and grasping space targets as an example, a lot of simulation and verification studies have been conducted for the algorithm proposed in this paper. Collision avoidance detecting algorithm is based on hierarchical structure rapid collision avoidance detection PQP package developed by OBB method and conducts the collision avoidance detection ${ }^{[18]}$ of humanoid robot motion planning. Fig.5 is the dynamic simulation effect for Armar III collision avoidance grasp targets. Fig.6 is configuration change track in C-space for Armar III collision avoidance grasp targets. The simulation result shows that when the sampling based on GIRRT algorithm can only make us know the initial configuration of 
humanoid robot but the goal configuration is unknown, the gradient project method can be applied to guide the single-direction RRT method and avoid the barriers and singularities of robot joints, it can effectively approach the goal target and plan the feasible and best path for the grasp object avoiding collision.

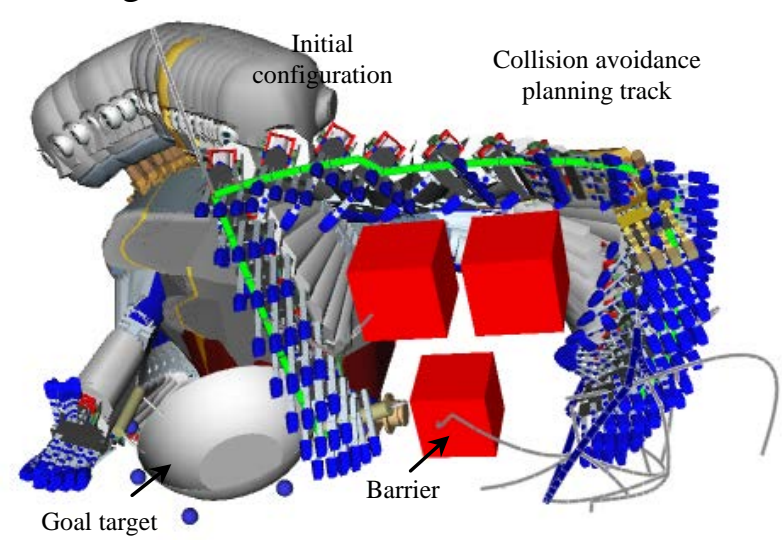

Fig.5 Dynamic simulation of humanoid robot Armar III collision avoidance grasp object

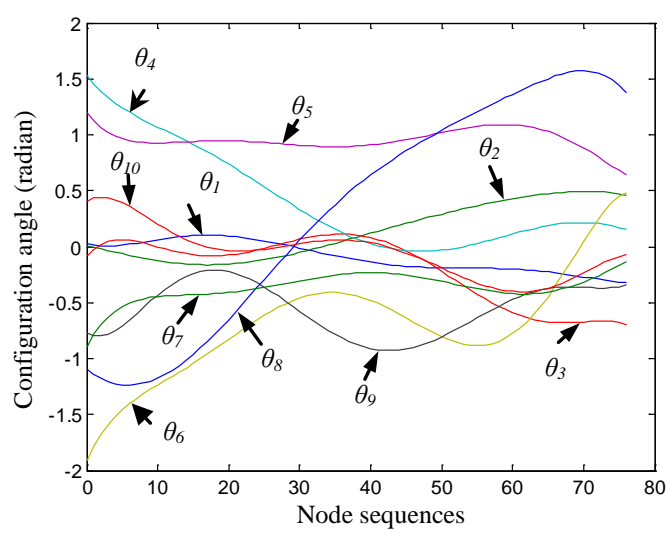

Fig.6 C-space configuration change tracks of Armar III collision avoidance grasp object

\section{Conclusion}

Grasp operation of humanoid robot is a key technology related to humanoid robot motion planning study. Aiming the grasp space limitation, redundant constrict and unknown goal configuration, this paper further proposes a weighted minimal norm GIRRT collision avoidance motion planning algorithm. On the one hand, it can fully make use of the completeness of rapidly-exploring random tree (RRT) method seeking paths. On the other hand, gradient projection method can be applied to seek the velocity optimal solution which avoids configuration singularity and joint constraint. Thus, the extension of humanoid root from initial configuration to goal target can be guided. Finally, a collision avoidance path can be found to complete the operation of arm grasp target. This method is of common significance in solving the grasp or manipulation of humanoid robot for dynamic goal or unknown goal position in a complex environment.

\section{Acknowledgement}

This work was sponsored by Shenzhen Science and Technology Program (JC201006020820A and JCYJ20120615101640639), Team of Scientific and Technological Innovation of Shenzhen Institute of Information Technology (CXTD2-002, LG2014005), Natural Science Foundation of Guangdong Province, China (S2013010013779), Guangdong Vocational Education Information Technology Fund (XXJS-2013-1019), School-enterprise Cooperation Projects(HX-054,HX-077).

\section{References}

[1] E. Yoshida, I. Belousov, C. Esteves, and J.-P. Laumond. Humanoid motion planning for dynamic tasks: Proceedings of the IEEE International Conference on Mechatronics \& Automation, 2005 [C]. Niagara Falls, Canada July 2005:1784-1789.

[2] L. Sentis and O. Khatib. Whole-body control framework for humanoids operating in human environments. Proceedings of International Conference on Robotics and Automation, 2006 [C]. 2006:2641-2648.

[3] O. Brock and L. E. Kavraki. Decomposition-based motion planning: A framework for real-time motion planning in high-dimensional configuration spaces. In Proceedings of International Conference on Robotics and Automation, 2001 [C]. 2001:1469-1474. 
[4] E. Drumwright and V. Ng-Thow-Hing. Toward interactive reaching in static environments for humanoid robots. In IEEE/RSJ International Conference On Intelligent Robots and Systems (IROS), 2006 [C]. Beijing, China, Oct 2006: 846-851.

[5] H.-L. Cheng, D. Hsu, J.-C. Latombe, and G. S'anchez-Ante. Multi-level free-space dilation for sampling narrow passages in PRM planning. In Proc. IEEE Int. Conf. on Robotics \& Automation, 2006 [C]. 2006:1255-1260.

[6]L. Jaillet and T. Siméon. A PRM-based motion planner for dynamically changing environments. In Proceedings of IEEE/RSJ International Conference on Intelligent Robots and Systems, 2004 [C]. Sendai, Japan, 2004:1606-1611.

[7] Z. Sun, D. Hsu, T. Jiang, H. Kurniawati, and J. H. Reif. Narrow passage sampling for probabilistic roadmap planning [J]. IEEE Transactions on Robotics and Automation, 2005, 21(6):1105-1115.

[8] K.I. Tsianos, I.A. Şucan, and L.E. Kavraki. Sampling-based robot motion planning: Towards realistic applications [J]. Computer Science Review, 2007,1 (1): 2-11.

[9] S La Valle, J Kuffner. Rapidly-exploring random trees: Progress and Prospects [A]. Proceedings of Algorithmic and Computational Robotics: New Directions, 2001 [C].2001:293-308.

[10] EGE E S. A new approach to increase performance of rapidly-exploring random trees (RRT) in mobile robotics. Proceedings of the IEEE International Conference on Robotics and Automation, April 17-19, 2006 [[C]]. Antalya. IEEE, 2006:851-855.

[11] Kang Liang, Zhao Chunxia and Guo Jianhui. Improved Path Planning Based on Rapidly-Exploring Random Tree for Mobile Robot in Unknown Environment [J]. Pattern Recognition and Artificial Intelligence, 2009, 22 (3): 337-343.

[12] J.J. Kuffner, K. Nishiwaki, S. Kagami, M. Inaba, and H. Inoue. Motion planning for humanoid robots. In Proceedings of $11^{\text {th }}$ International Symposium of Robotics Research, 2003 [C].2003:365-374.

[13] J.J. Kuffner, S. Kagami, K. Nishiwaki, M. Inaba, and H. Inoue. Dynamically stable motion planning for humanoid robots [J]. Autonomous Robots, 2002, 12 (1): pp. 105-118.

[14] D. Bertram, J.J. Kuffner, R. Dillmann and T. Asfour. An integrated approach to inverse kinematics and path planning for redundant manipulators. In Proceedings of IEEE International Conference on Robotics and Automation, 2006 [C].2006:1874-1879.

[15] M.K. Weghe, D. Ferguson, and S.S. Srinivasa. Randomized path planning for redundant manipulators without inverse kinematics. In Proceedings of IEEE International Conference on Humanoid Robots, 2007 [C]. 2007:477-482.

[16] D. Berenson, S.S. Srinivasa, D. Ferguson, and J.J. Kuffner. Manipulation planning on constraint manifolds. In Proceedings of IEEE International Conference on Robotics and Automation, 2009 [C]. 2009:625-632.

[17] L. Guilamo, J. Kuffner, K. Nishiwaki, and S. Kagami. Efficient prioritized inverse kinematic solutions for redundant manipulators. IEEE/RSJ International Conference on Intelligent Robots and Systems, 2005 [C]. 2005:3921-3926.

[18] S. Gottschalk, M.C. Lin and D. Manocha. OBB Tree: A hierarchical structure for rapid interference detection. In Proceedings of SIGGRAPH 96', 1996 [C]. 1996:171-180. 